\title{
Overexpression of microRNA-495 suppresses the proliferation and invasion and induces the apoptosis of osteosarcoma cells by targeting high-mobility group nucleosome-binding domain 5
}

\author{
WEIBO JIANG $^{1}$, JIA ZHENG ${ }^{2}$, TAO YU ${ }^{1}$ and JINCHENG WANG ${ }^{1}$ \\ ${ }^{1}$ Department of Orthopaedics, The Second Hospital of Jilin University, Changchun, Jilin 130041; \\ ${ }^{2}$ Department of Radiology, China-Japan Union Hospital of Jilin University, Changchun, Jilin 130031, P.R. China
}

Received November 7, 2016; Accepted December 27, 2016

DOI: $10.3892 / o r .2017 .5715$

\begin{abstract}
It has been suggested that microRNAs (miRNAs) act as critical regulators in tumorigenesis. MicroRNA-495 (miR-495) has been suggested as a cancer-associated miRNA in various types of cancers; however, the role of miR-495 in osteosarcoma is unknown. The aim of the present study was to determine whether miR-495 is involved in osteosarcoma, and to investigate the potential molecular mechanism of its involvement. We found that miR-495 was significantly downregulated in osteosarcoma tissues and cell lines, as detected by real-time quantitative polymerase chain reaction (RT-qPCR). Overexpression of miR-495 inhibited osteosarcoma cell proliferation in 3-(4,5-dimethylthiazol2-yl)-2,5-diphenyltetrazolium bromide, colony formation and cell cycle assays. Overexpression of miR-495 induced osteosarcoma cell apoptosis. Moreover, miR-495 overexpression also inhibited osteosarcoma cell invasion. Bioinformatics and luciferase reporter assays demonstrated that miR-495 targets the 3'-untranslated region of high-mobility group nucleosome-binding domain 5 (HMGN5), a potential oncogene in various types of cancers. Overexpression of miR- 495 inhibited the expression of HMGN5, cyclin B1, Bcl-2 and matrix metalloproteinase 9. In addition, restoration of HMGN5 protein expression abrogated the miR-495-induced effects. Taken together, the present study indicated that miR-495 suppresses the proliferation and invasion and induces the apoptosis of osteosarcoma cells by targeting HMGN5, providing a novel
\end{abstract}

Correspondence to: Professor Jincheng Wang, Department of Orthopaedics, The Second Hospital of Jilin University, 218 Ziqiang Street, Changchun, Jilin 130041, P.R. China

E-mail: wang_jinchengjc@163.com

Abbreviations: miRNAs, microRNAs; RT-qPCR, real-time quantitative polymerase chain reaction; MTT,3-(4,5-dimethylthiazol2-yl)-2,5-diphenyltetrazolium bromide; UTR, untranslated region; HMGN5, high-mobility group nucleosome-binding domain 5; MMP9, matrix metalloproteinase 9

Key words: osteosarcoma, miR-495, HMGN5, proliferation, apoptosis, invasion insight into the molecular pathogenesis of osteosarcoma and suggesting a potential molecular target for the development of an miRNA-targeted therapeutic strategy for osteosarcoma.

\section{Introduction}

Osteosarcoma is the most common bone malignancy among patients in the pediatric age group (1). Although therapeutic interventions for osteosarcoma have improved over the past decade, the 5-year overall survival rate is still low $(2,3)$. The molecular pathogenesis of osteosarcoma remains unclear, which has hampered efforts to improve treatment. Therefore, a better understanding of the molecular pathogenesis of osteosarcoma is crucial for the development of effective therapies for this disease.

MicroRNAs (miRNAs) are a class of endogenous small RNAs of 22 nucleotides in length, and they negatively modulate gene expression by targeting the 3 '-untranslated region (3'-UTR) of mRNAs, leading to translational inhibition $(4,5)$. By post-transcriptionally regulating target genes, miRNAs are involved in various cellular processes, including cell proliferation, apoptosis, migration and invasion (6). In recent years, a growing body of evidence suggests that miRNAs with deregulated expression patterns are critical regulators in tumorigenesis (7-9). Various miRNAs have been found to participate in the pathogenesis of osteosarcoma, regulating numerous target genes and critical pathways (10-12). These deregulated miRNAs have the potential to serve as biomarkers for diagnosis and prognosis (10-12). Targeting miRNAs has also shown promise for the treatment of osteosarcoma. A better understanding of the role of miRNAs in osteosarcoma may aid in the development of miRNA-targeted therapies.

High-mobility group nucleosome-binding domain 5 (HMGN5), also known as nucleosome-binding protein 1, has been suggested as an potential oncogene in several types of cancers $(13,14)$. HMGN5 can regulate histone modification, DNA replication, DNA repair, and gene transcription through binding to chromatin regulators (15). HMGN5 is found in a variety of tissues $(16,17)$, and regulates the expression of numerous genes (18). However, dysregulation of HMGN5 is associated with cancer progression and development (13). To date, high expression of HMGN5 has been linked to many 
types of cancers including gliomas (19), prostate cancer (20), renal cell carcinoma (21), breast (22) and bladder cancer (23). Furthermore, overexpression of HMGN5 is also found in cases of osteosarcoma that are associated with cell proliferation, metastasis, and drug resistance (24). HMGN5 has the potential to serve as a molecular target for the prevention and treatment of osteosarcoma.

MicroRNA-495 (miR-495) is associated with tumorigenesis in various types of cancers, through the regulation of numerous target genes (25-28). It may be also involved in osteosarcoma. However, no data have yet been reported. The aim of the present study was to determine whether miR-495 is involved in osteosarcoma, and to investigate the potential molecular mechanism of its involvement. We found that miR-495 was significantly downregulated in osteosarcoma tissues and cell lines. Overexpression of miR-495 inhibited proliferation, induced apoptosis and repressed the invasion of osteosarcoma cells. Notably, we identified HMGN5 as a potential target gene of miR-495 in osteosarcoma cells. miR-495 also regulated the expression of cyclin B1, Bcl-2 and matrix metalloproteinase 9 (MMP9) which are the downstream targets of HMGN5 involved in regulating cancer cell proliferation, apoptosis and invasion $(20,21,23)$. The restoration of HMGN5 abrogated the miR-495-induced antitumor effects. Taken together, our findings indicate that miR-495 exerts antitumor effects in osteosarcoma by targeting HMGN5, providing novel insight into the molecular pathogenesis of osteosarcoma and suggesting a potential molecular target for the development of an miRNA-targeted therapeutic strategy for osteosarcoma.

\section{Materials and methods}

Clinical samples. Fifteen paired human osteosarcoma and adjacent normal tissues (located $>3 \mathrm{~cm}$ from the tumor) were obtained from the Department of Orthopaedics at The Second Hospital of Jilin University. The resected tissues were immediately frozen in liquid nitrogen and stored at $-80^{\circ} \mathrm{C}$ for use. Use of the clinical tissue samples was approved by the Institutional Human Experiment and Ethics Committee of The Second Hospital of Jilin University, with written informed consent from all of the participants.

Cell lines. Human osteosarcoma cell lines (143B, SaOS-2, U2OS and MG63), normal osteoblastic cell line hFOB and 293T cells were purchased from the American Type Culture Collection (ATCC; Manassas, VA, USA). Cells were cultured in Dulbecco's modified Eagle's medium (DMEM; Invitrogen, Carlsbad, CA, USA), supplemented with $10 \%$ fetal bovine serum (FBS) and 1\% penicillin-streptomycin solution (both from Gibco, Rockville, MD, USA), and maintained in a humidified incubator containing $5 \% \mathrm{CO}_{2}$ at $37^{\circ} \mathrm{C}$.

RNA extraction and real-time quantitative polymerase chain reaction $(R T-q P C R)$. Total RNA was extracted using TRIzol reagent (Invitrogen), according to the recommended protocols. The RNA samples were pretreated with DNase I (Takara, Dalian, China) before cDNA synthesis. To detect HMGN5 mRNA expression, cDNA was generated by Moloney murine leukemia virus (M-MLV) reverse transcriptase (BioTeke, Corporation, Beijing, China). To detect miR-495 expression,
cDNA was generated with the TaqMan MicroRNA reverse transcription kit (Applied Biosystems, Foster City, CA, USA). RT-qPCR was conducted with SYBR-Green PCR Master Mix with a 7900HT Fast Real-Time PCR System (both from Applied Biosystems). U6 and glyceraldehyde-3-phosphate dehydrogenase (GAPDH) were used for normalization. Relative gene expression was calculated by the $2^{-\Delta \Delta C t}$ method. The fold-change of gene expression was obtained by normalization against the control group. The primer sequences were as follows: miR-495, 5'-AAACAAACAUGGUGCACUU CUU-3' and 5'-GAAGUGCACCAUGUUUGUUUUU-3'; U6, 5'-GCTTCGGCAGCACATATACTAAAAT-3' (forward) and 5'-CGCTTCACGAATTTGCGTGTCAT-3' (reverse); HMGN5, 5'-GCAGTCAGGCAGTGACTGCCTTCG-3' (forward) and 5'-CCCTTTTCTGTGGCATCTTC-3' (reverse); and GAPDH, 5'-TGTGTCCGTCGTGGATCTGA-3' (forward) and 5'-TTGCTGTTGAAGTCGCAGGAG-3' (reverse).

Cell transfection. The miR-495 mimics and negative control (miR-NC) were purchased from RiboBio (Guangzhou, China) and transfected into cells using Lipofectamine 2000 (Invitrogen) following the manufacturer's instructions. The HMGN5 cDNA without 3'-UTR was cloned into a pcDNA3.1 vector (RiboBio) to generate a pcDNA3.1/HMGN5 overexpressing vector. The vector was transfected into cells by Lipofectamine 2000. The transfection efficacy was assessed using RT-qPCR or western blot analysis after a 48-h transfection period.

MTT assay. Cells were seeded into 96-well plates at a density of $5 \times 10^{3}$ cells/well and cultured overnight. Cells were transfected with either miR-495 mimics or miR-NC for $48 \mathrm{~h}$. Then, the medium was refreshed and $20 \mu \mathrm{l}$ of 3-(4,5-dimethylthiazol-2-yl)-2,5-diphenyltetrazolium bromide (MTT; $5 \mathrm{mg} / \mathrm{ml}$; Sigma-Aldrich, St. Louis, MO, USA) was added to each well. The cells were cultured for $4 \mathrm{~h}$ and $200 \mu \mathrm{l}$ of dimethyl sulfoxide (DMSO; Sigma-Aldrich) was added to dissolve the formazan crystals. After $15 \mathrm{~min}$, the absorbance was detected with a wavelength of $490 \mathrm{~nm}$ by an ELISA reader (Bio-Rad, Hercules, CA, USA).

Colony formation assay. The transfected cells were plated into 6-well plates at a density of 100 cells/well and cultured in growth medium containing $0.3 \%$ noble agar (Sigma-Aldrich) for 10 days. Afterwards, the colonies were stained with $1 \%$ crystal violet (Sigma-Aldrich). The colonies were counted under a microscope (Olympus, Tokyo, Japan) and analyzed.

Cell cycle assay. After treatment, cells were fixed with $70 \%$ ethanol and washed with phosphate-buffered saline (PBS). Cells were then treated with $100 \mu \mathrm{g} / \mathrm{ml}$ of propidium iodide (PI; Sigma-Aldrich) and $10 \mu \mathrm{g} / \mathrm{ml}$ of RNase A (Roche Applied Science, Indianapolis, IN, USA) for $30 \mathrm{~min}$ at room temperature in a dark place. Cells were examined by flow cytometer BD FACSCalibur (BD Biosciences, San Jose, CA, USA). The data were collected by CellQuest software (BD Biosciences).

Annexin V/PI apoptosis assay. Cell apoptosis was detected using an Annexin V/PI double staining kit (Beyotime Biotechnology, Haimen, China). Cells were harvested and 

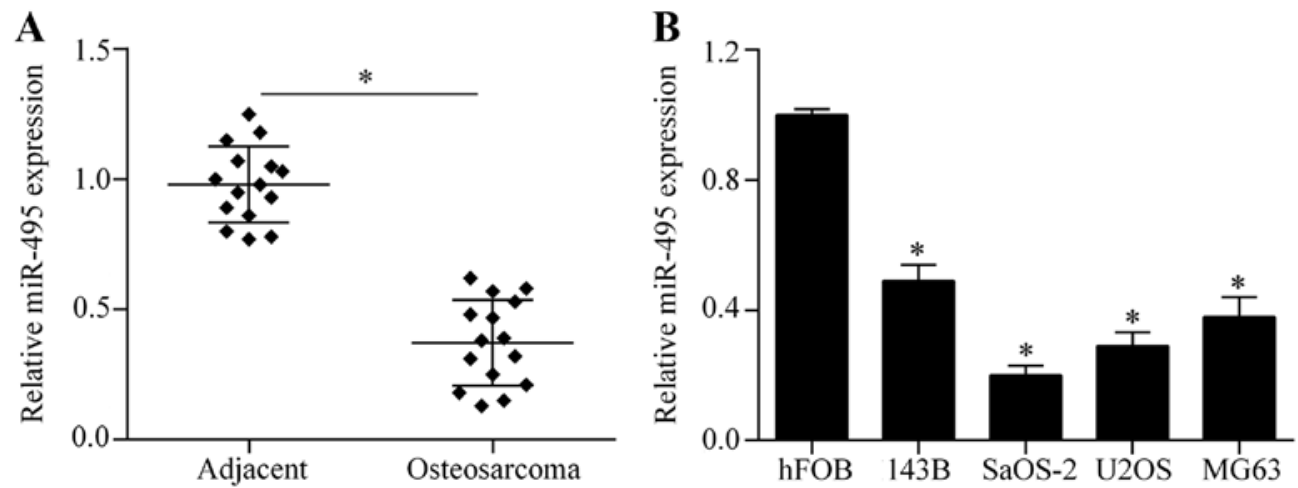

Figure 1. Expression of miR-495 in osteosarcoma. (A) Expression of miR-495 in osteosarcoma and adjacent normal tissues ( $\mathrm{n}=15)$ was detected using RT-qPCR; "p<0.05. (B) Expression of miR-495 in 143B, SaOS-2, U2OS, MG63 and hFOB cells was detected by RT-qPCR; "p<0.05 vs. hFOB.

digested with $2.5 \mathrm{~g} / 1$ of trypsin. After washing with PBS, cells were re-suspended in $200 \mu \mathrm{l}$ of binding buffer, and treated with $10 \mu \mathrm{l}$ of Annexin $\mathrm{V}$ for 30 min followed by incubation with $5 \mu$ of PI solution for 5 min in a dark place. Then, cells were examined by flow cytometry (BD Biosciences).

Caspase-3 activity assay. Caspase-3 activity was detected using a commercial kit (Roche Applied Science), following the manufacturer's recommended protocols. Cells were lysed and incubated with DEVD-pNA substrate at $37^{\circ} \mathrm{C}$ for $2 \mathrm{~h}$. The absorbance at $405 \mathrm{~nm}$ was determined using an ELISA reader (Bio-Rad).

Invasion assay. Cell invasion was detected using Transwell assay. The upper chamber of the Transwell inserts (Costar, Corning, NY, USA) was precoated with Matrigel (BD Biosciences). After transfection, $1 \times 10^{5}$ cells were re-suspended in $500 \mu \mathrm{l}$ of serum-free medium, and added to the upper chamber. Meanwhile, the lower chamber was filled with $500 \mu \mathrm{l}$ of growth medium containing 10\% FBS. The cells were cultured at $37^{\circ} \mathrm{C}$ for $24 \mathrm{~h}$. Afterwards, the filters were removed, fixed with $20 \%$ methanol, and stained with $1 \%$ crystal violet (Sigma-Aldrich). The invaded cells were counted under a microscope (Olympus).

Western blot analysis. Proteins were extracted using lysis buffer (Beyotime Biotechnology), and equivalent amounts of proteins were loaded on $12.5 \%$ sodium dodecyl sulfate polyacrylamide gels for separation, and then, transferred to a nitrocellulose membrane (Bio-Rad). After being blocked with $3 \%$ non-fat milk, the membrane was blotted with primary antibodies at $4^{\circ} \mathrm{C}$ overnight, and then incubated with horseradish peroxidaseconjugated secondary antibodies (1:2,000; Bioss, Beijing, China). The protein bands were developed using enhanced chemiluminescence (Millipore, Boston, MA, USA). The intensity of the protein bands was detected using Image-Pro Plus 6.0 software (Media Cybernetics, Inc., Rockville, MD, USA). The fold-change of protein expression was obtained by normalization with GAPDH, and then compared with the control group. The primary antibodies, including anti-HMGN5, anti-cyclin B1, anti-Bcl-2, anti-MMP9 and anti-GAPDH, were purchased from Santa Cruz Biotechnology (Santa Cruz, CA, USA).
Luciferase reporter assays. HMGN5-3'-UTR containing either the predicted seed sequence of miR-495 or a mutated binding site of the 3'-UTR were inserted into a pmirGLO Dual-Luciferase plasmid (Promega, Madison, WI, USA). The recombinant vector was then cotransfected with either miR-495 mimics or miR-NC, into 293T cells using Lipofectamine 2000. After $48 \mathrm{~h}$, the relative luciferase activity was analyzed using the Dual-Luciferase Assay kit (Promega).

Data analysis. Data are expressed as means \pm standard deviation. Statistical analyses were performed using the SPSS package (version 11.5; SPSS, Inc., Chicago, IL, USA). Differences were analyzed using Student's t-test or one-way analysis of variance followed by Bonferroni's post hoc test. Values of $p<0.05$ were regarded as statistically significant.

\section{Results}

The expression of miR-495 is downregulated in osteosarcoma tissues and cell lines. To investigate the possible role of miR-495 in osteosarcoma, we first investigated the expression of miR-495 in osteosarcoma tissues by RT-qPCR. The results showed that miR-495 was significantly downregulated in osteosarcoma tissues compared to corresponding adjacent normal tissues (Fig. 1A). Next, we further examined the expression pattern of miR-495 in four osteosarcoma cell lines: 143B, SaOS-2, U2OS and MG63. We found that miR-495 was markedly decreased in the osteosarcoma cell lines compared with the normal osteoblastic cell line, hFOB (Fig. 1B). Taken together, these findings indicate that miR-495 acts in a tumor-suppressing role in cases of osteosarcoma.

Overexpression of miR-495 inhibits osteosarcoma cell proliferation. To investigate whether miR-495 shows an antitumor effect on osteosarcoma cells, we overexpressed miR-495 in osteosarcoma by transiently transfecting miR-495 mimics into SaOS-2 and U2OS cells (Fig. 2A). The effect of miR-495 overexpression on cell proliferation was detected by MTT assay. The results showed that overexpression of miR-495 significantly inhibited osteosarcoma cell proliferation (Fig. 2B). Colony formation assay showed that overexpression of miR-495 significantly suppressed the colony-forming capacity of the osteosarcoma cells (Fig. 3A). 

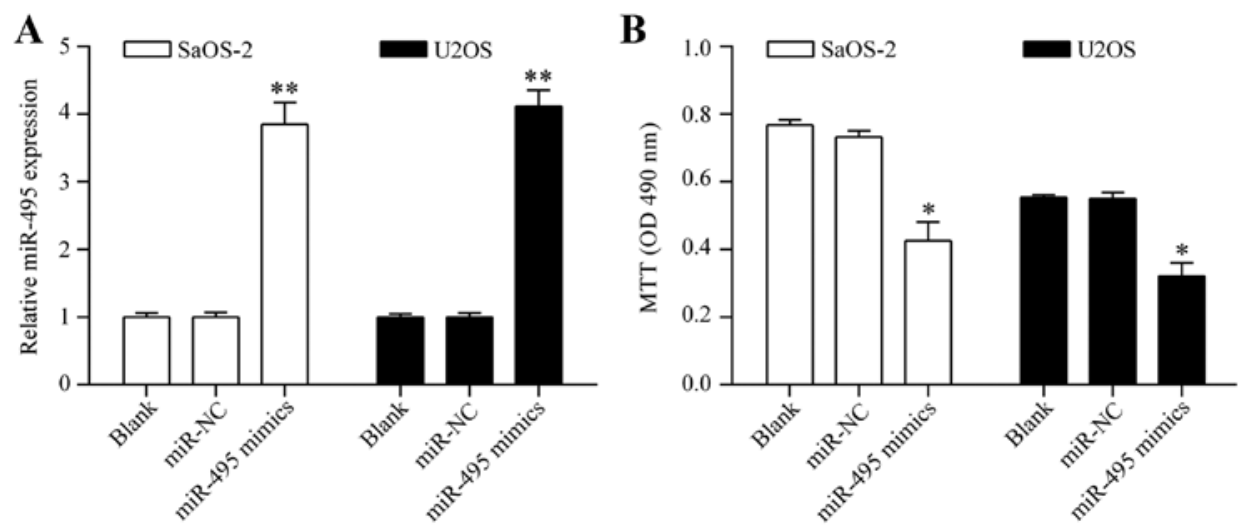

Figure 2. miR-495 inhibits osteosarcoma cell proliferation. SaOS-2 and U2OS cells were transfected with miR-495 mimics or miR-NC for $48 \mathrm{~h}$. (A) Expression of miR-495 was detected using qRT-PCR; ${ }^{* *} \mathrm{p}<0.01$ vs. blank and miR-NC. (B) Cell proliferation was detected by MTT assay; ${ }^{*} \mathrm{p}<0.05$ vs. blank and miR-NC.
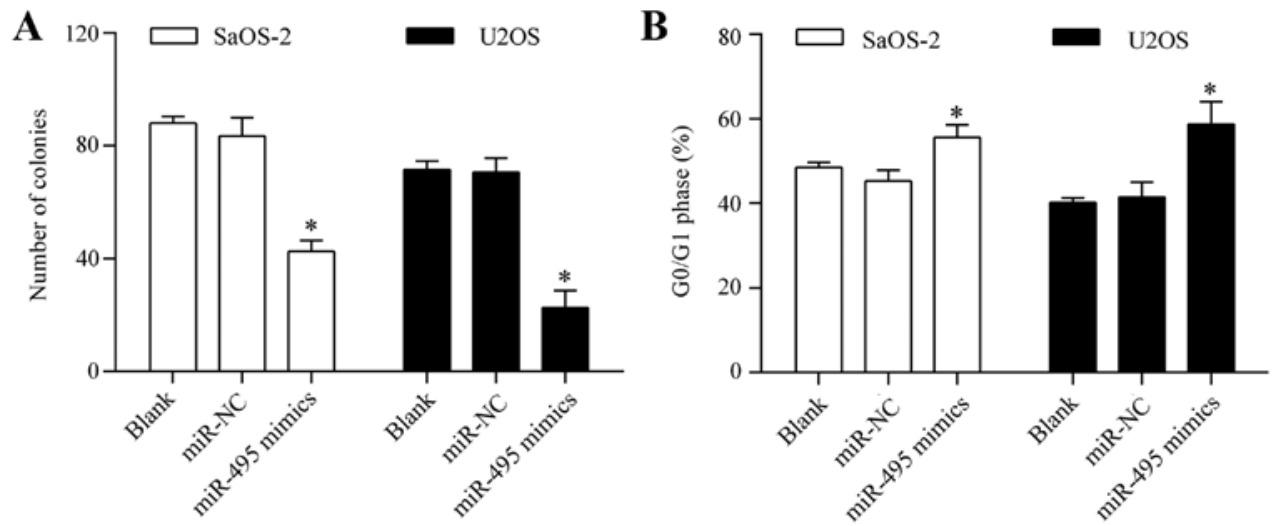

Figure 3. miR-495 suppresses colony formation and induces G0/G1 cell cycle arrest. (A) Colony formation assay of the effect of miR-495 overexpression on the colony-forming capacity of osteosarcoma cells. SaOS-2 and U2OS cells were transfected with miR-495 mimics or miR-NC for $48 \mathrm{~h}$, and then subjected to colony formation for 10 days. (B) Cell cycle analysis of miR-495-overexpressing cells in the G0/G1 phase. SaOS-2 and U2OS cells were transfected with miR-495 mimics or miR-NC for $48 \mathrm{~h}$, and the percentage of G0/G1 phase cells was then detected using flow cytometric assay; ${ }^{\text {* }}<<0.05 \mathrm{vs}$. blank and miR-NC.
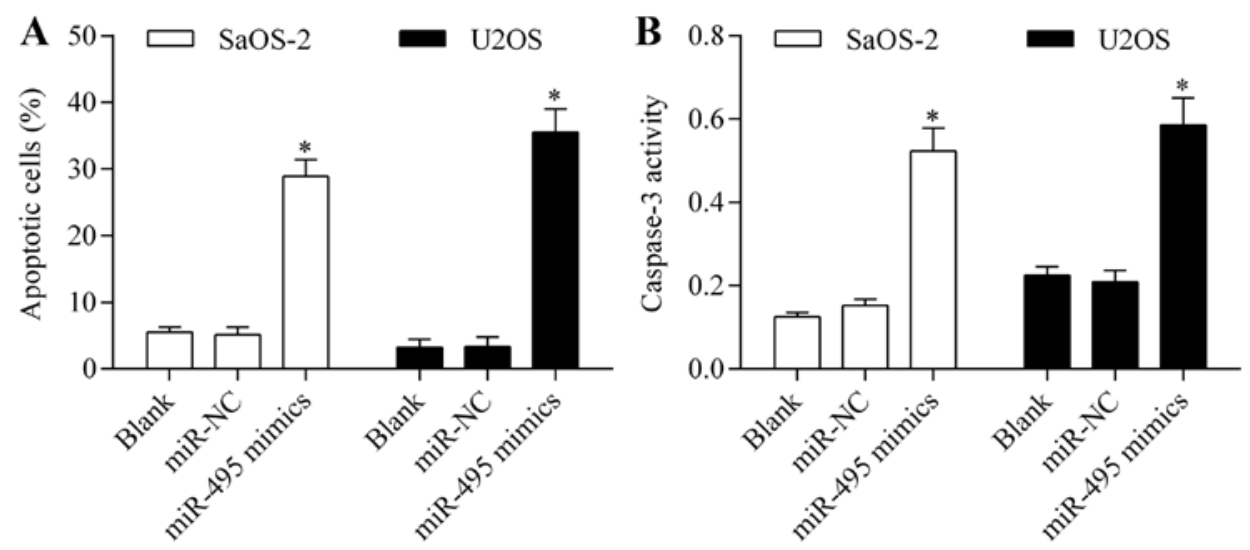

Figure 4. miR-495 induces osteosarcoma cell apoptosis. SaOS-2 and U2OS cells were transfected with miR-495 mimics or miR-NC for $48 \mathrm{~h}$. Cell apoptosis was detected by (A) Annexin V/PI apoptosis and (B) caspase-3 activity assays; ${ }^{*} \mathrm{p}<0.05$ vs. blank and miR-NC.

Analysis of the cell cycle distribution showed that miR-495 overexpression markedly induced cell cycle arrest in the G0/G1 phase (Fig. 3B). Overall, these results suggest that miR-495 inhibits osteosarcoma cell proliferation.

Overexpression of miR-495 induces osteosarcoma cell apoptosis. To confirm the antitumor effect of miR-495 on osteosarcoma cells, we examined the effect of miR-495 overexpression on cell apoptosis. Annexin V/PI apoptosis assay showed that overexpression of miR-495 significantly promoted osteosarcoma cell apoptosis (Fig. 4A). Moreover, miR-495 overexpression markedly increased the activity of caspase-3 (Fig. 4B). These data imply that miR-495 induces osteosarcoma cell apoptosis. 


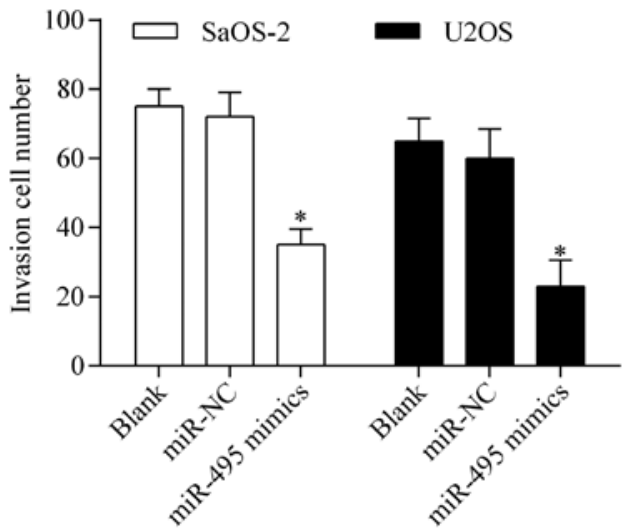

Figure 5. miR-495 suppresses osteosarcoma cell invasion. SaOS-2 and U2OS cells were transfected with miR-495 mimics or miR-NC for $48 \mathrm{~h}$, and then subjected to Transwell invasion assay; ${ }^{*} \mathrm{p}<0.05$ vs. blank and miR-NC.

Overexpression of miR-495 suppresses osteosarcoma cell invasion. To further investigate the antitumor effect of miR-495, we detected the effect of miR-495 overexpression on osteosarcoma cell invasion by Transwell invasion assay. The results showed that overexpression of miR-495 significantly repressed the invasive potential of SaOS-2 and U2OS cells (Fig. 5), indicating that miR-495 inhibits osteosarcoma cell invasion.

HMGN5 is the potential target gene of miR-495 in osteosarcoma cells. To investigate the underlying mechanism by which miR-495 exerts an antitumor effect, we performed bioinformatics analysis to identify the target gene of miR-495. HMGN5, a well-known oncogene associated with osteosarcoma tumorigenesis (13), was predicted as the target gene of miR-495. The putative binding sites of miR-495 within the wild-type 3'-UTR of HMGN5 are described in Fig. 6A. The complementary seed sequences were mutated to generate the mutant 3'-UTR of HMGN5 (Fig. 6A). To confirm the interaction between miR-495 and HMGN5 3'-UTR, dual-luciferase assay was performed using pmirGLO dual-luciferase containing either the wild-type 3'-UTR of HMGN5 or the mutant 3'-UTR of HMGN5. The results showed that the luciferase activity of the reporter vector with the wild-type 3'-UTR of HMGN5 was significantly suppressed by miR-495 overexpression (Fig. 6B). However, miR-495 overexpression showed no significant effect on luciferase activity of the reporter vector with the mutant 3'-UTR of HMGN5 (Fig. 6B), indicating that miR-495 directly targeted the 3'-UTR of HMGN5. To further verify that HMGN5 is the target gene of miR-495, we examined the effect of miR-495 on HMGN5 expression. The results showed that HMGN5 mRNA (Fig. 7A) and protein (Fig. 7B) expression levels were significantly reduced by miR-495 overexpression. Taken together, these results suggest that HMGN5 is the potential target gene of miR-495.

Overexpression of miR-495 affects the downstream genes of HMGN5. To further investigate the molecular mechanism of miR-495-induced antitumor effects, we detected the effect of miR-495 on cyclin B1, Bcl-2 and MMP9 expression which are the characterized downstream genes of HMGN5 $(20,21,23)$. The results showed that the protein expression levels of cyclin B1, Bcl-2 and MMP9 (Fig. 8A-D) were significantly decreased by miR- 495 overexpression. These results indicate that miR-495-induced antitumor effects are associated with the inhibition of cyclin B1, Bcl-2 and MMP9.

HMGN5 is involved in miR-495-induced antitumor effects. To validate whether miR-495 exerts antitumor effects through HMGN5, we performed rescue experiments using pcDNA3.1/HMGN5 as an overexpressing vector. The results showed that the decreased HMGN5 expression induced by miR-495 overexpression was markedly restored by transfection of the pcDNA3.1/HMGN5 vector (Fig. 9A). We then evaluated the effect of HMGN5 restoration on miR-495-induced antitumor effects. The results showed that HMGN5 restoration
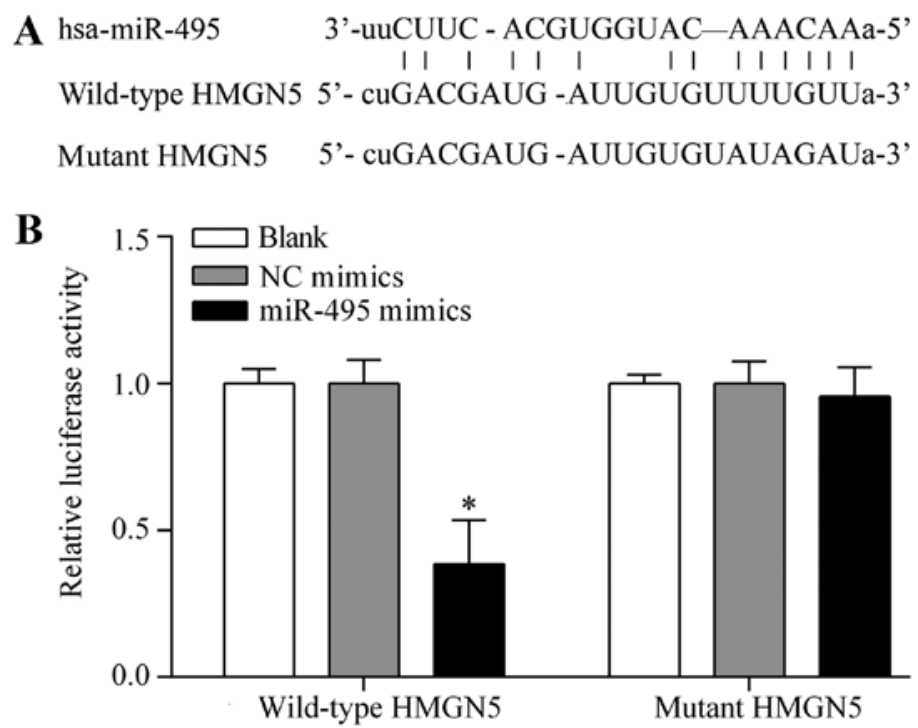

Figure 6. miR-495 targets the 3'-UTR of HMGN5. (A) Schematic diagram of the miR-495 binding sites and mutant binding sites in the 3'-UTR of HMGN5. (B) Dual-luciferase reporter assays showed the effects of miR-495 overexpression on luciferase activity. pmirGLO dual-luciferase vector containing wild-type or mutant 3'-UTR of HMGN5 were cotransfected with miR-495 mimics or miR-NC into 293T cells for $48 \mathrm{~h}$; " $\mathrm{p}<0.05 \mathrm{vs}$. blank and miR-NC. 
$\mathbf{A}$

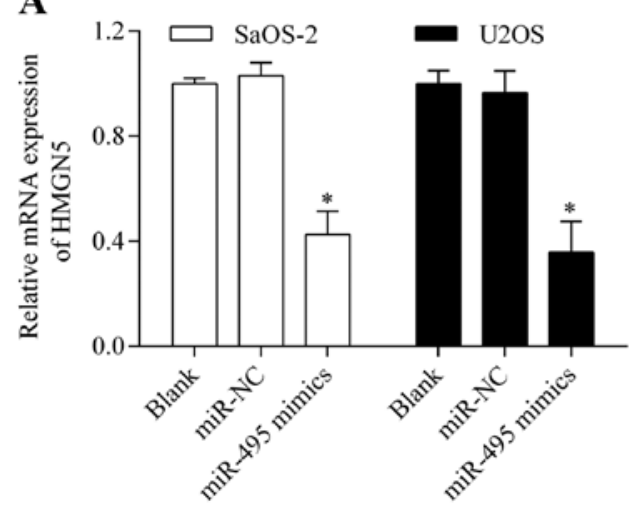

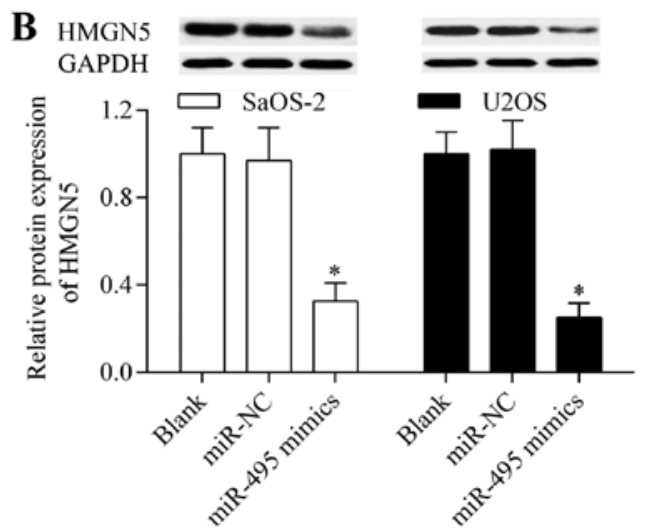

Figure 7. miR-495 inhibits HMGN5 expression. SaOS-2 and U2OS cells were transfected with miR-495 mimics or miR-NC for 48 h. (A) The mRNA and (B) protein expression levels of HMGN5 were detected by RT-qPCR and western blotting, respectively; " $\mathrm{p}<0.05 \mathrm{vs}$. blank and miR-NC.

A

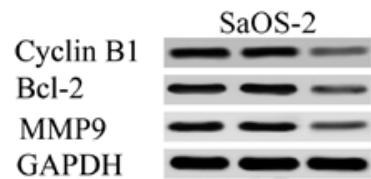

B

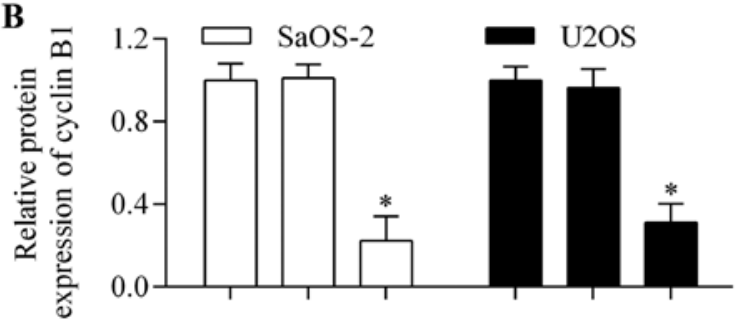

C
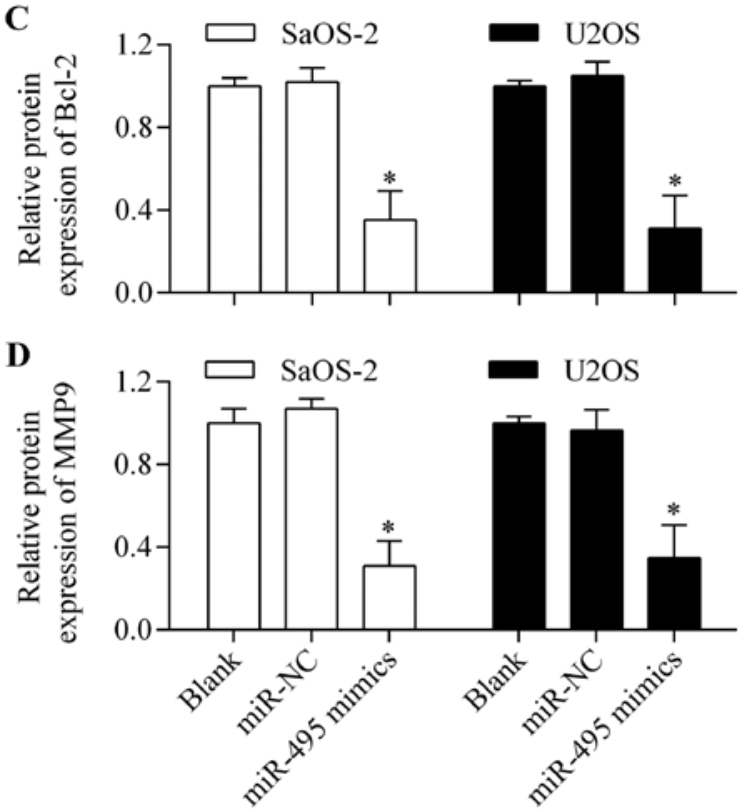

Figure 8. miR-495 inhibits the expression of cyclin B1, Bcl-2 and MMP9. SaOS-2 and U2OS cells were transfected with miR-495 mimics or miR-NC for $48 \mathrm{~h}$. (A) Protein expression levels of cyclin B1, Bcl-2 and MMP9 were detected by western blot analysis. Relative protein expression of (B) cyclin B1, (C) Bcl-2 and (D) MMP9 was quantitatively analyzed using Image-Pro Plus $6.0 ;{ }^{*} \mathrm{p}<0.05$ vs. blank and miR-NC.

significantly reversed the effects of miR-495 overexpression on cell proliferation (Fig. 9B), apoptosis (Fig. 9C) and inva- sion (Fig. 9D). Overall, these findings indicate that miR-495 exerts antitumor effects by targeting and inhibiting HMGN5.

\section{Discussion}

A growing body of evidence suggests that miRNAs are critical regulators in osteosarcoma (12). Deregulated miRNAs can be used as biomarkers for the diagnosis and prognosis of cancer (10-12). Targeting miRNAs has shown promise for the treatment of osteosarcoma. Therefore, it is of great importance to gain a better understanding of how miRNAs act in osteosarcoma. In the present study, we revealed that miR-495 is a novel miRNA involved in osteosarcoma. We found that miR-495 was downregulated in osteosarcoma and that overexpression of miR-495 inhibited osteosarcoma cell proliferation and invasion and promoted cell apoptosis. These findings suggest that miR-495 may be associated with the development and progression of osteosarcoma, and could be a potential molecular target for the development of miRNA-targeted therapies.

The deregulated expression of miR-495 has been found in numerous types of cancers (29-32). Li et al reported that miR-495 inhibited gastric cancer cell migration and invasion through targeting phosphatase of regenerating liver-3 (33). Similarly, gastric cancer cell migration and invasion can be suppressed though demethylation treatment, which upregulates miR-495 (34). Numerous studies have reported that miR-495 is decreased in brain tumors and can be used to inhibit tumor progression by targeting cyclin-dependent kinase 6 (35), Glut1 (36), Gfil (37) and v-myb avian myeloblastosis viral oncogene homolog (38). Downregulation of miR-495 was found in non-small cell lung cancer tissues and cells, and overexpression of miR-495 inhibited cell proliferation and migration by targeting metastasis-associated protein 3 (25). Moreover, overexpression of miR-495 promoted the sensitivity of non-small cell lung cancer cells to platinum by inhibiting copper-transporting P-type adenosine triphosphatase A (39). $\mathrm{Xu}$ et al reported that miR-495 inhibited cell growth and migration in endometrial cancer by targeting Forkhead box $\mathrm{C} 1$ (40). Overexpression of miR-495 also inhibited prostate cancer cell migration and invasion by targeting Akt and the mammalian target of rapamycin signaling (28). Additionally, miR-495 induced cell cycle arrest in breast cancer cells by 

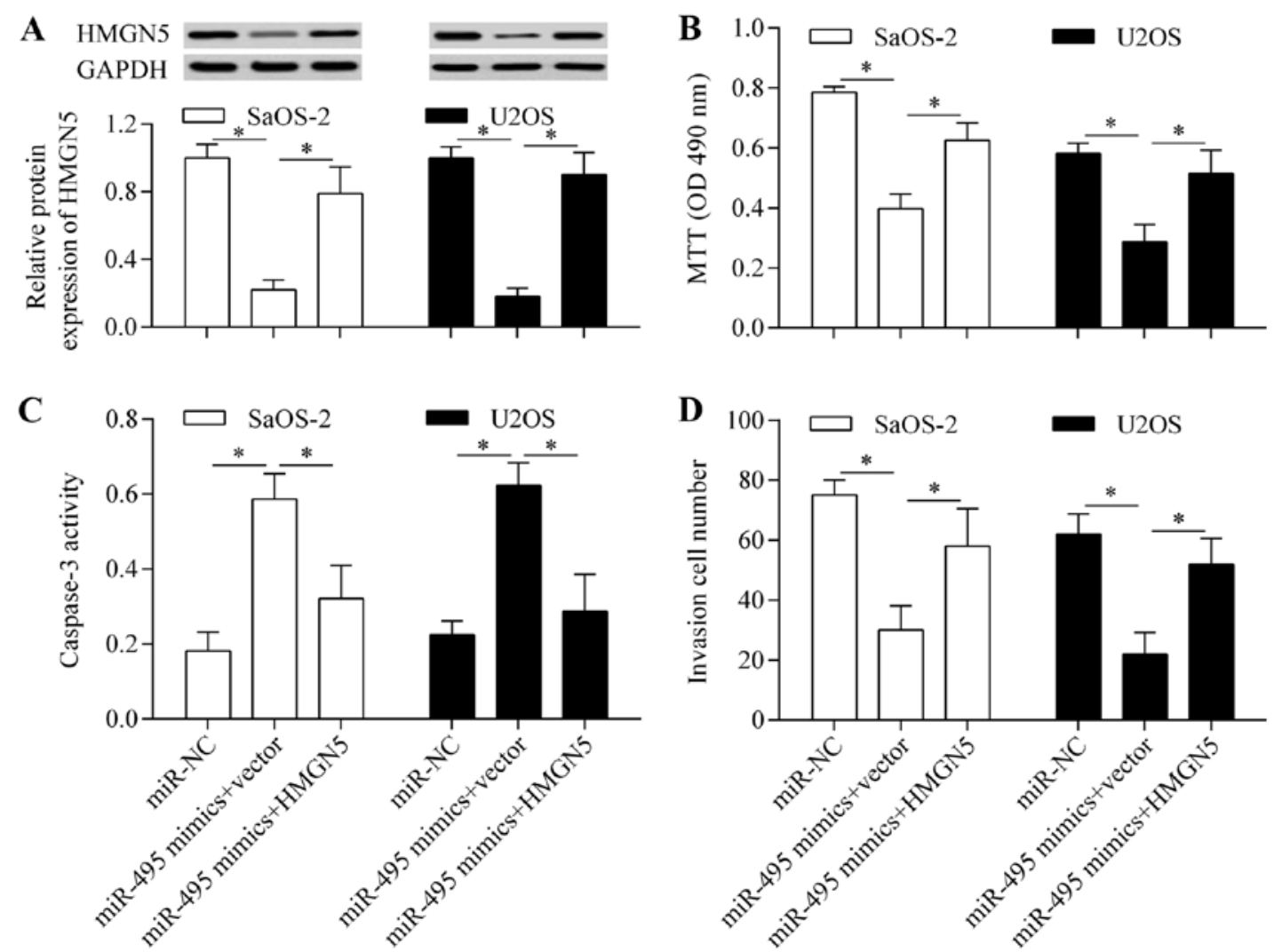

Figure 9. Restoration of HMGN5 abolishes the miR-495-induced antitumor effects. SaOS-2 and U2OS cells were cotransfected with miR-495 mimics and pcDNA3.1/HMGN5 vector and cultured for 48 h. miR-495 mimics + vector, cells were cotransfected with miR-495 mimics and pDNA3.1 empty vector; miR-495 mimics + HMGN5, cells were cotransfected with miR-495 mimics and pcDNA3.1/HMGN5 vector. (A) Protein expression level of HMGN5 was detected by western blotting. (B) Cell proliferation was determined by MTT assays. (C) Cell apoptosis was detected by caspase-3 activity assay. (D) Cell invasion was examined using Transwell invasion assays; ${ }^{*} \mathrm{p}<0.05$.

inhibiting B cell-specific Moloney murine leukemia virus integration site 1 (27). Overall, these findings suggest that miR-495 has a tumor suppressive role. However, an oncogenic role of miR-495 has also been reported. In breast cancer stem cells, miR-495 was found to be overexpressed, and promoted cell proliferation and invasion in hypoxia (41). In addition, overexpression of miR-495 induced breast cancer cell migration by targeting junctional adhesion molecule A (26). Under hypoxic conditions, miR-495 was found to be overexpressed and promote proliferation and tumor angiogenesis of gastric cancer cells by inhibiting Runt-related transcription factor 3 (42). In the present study, we found that miR-495 was significantly decreased in osteosarcoma tissues and cell lines. Overexpression of miR-495 suppressed osteosarcoma cell proliferation and invasion and induced cell apoptosis of osteosarcoma cells, supporting a tumor-suppressive role of miR-495 in osteosarcoma. The apparent discrepancy in the role of miR-495 in tumorigenesis implies that the precise biological role of miR-495 may be dependent on cell type and condition. Thus, the role of miR-495 in tumorigenesis requires further investigation.

To investigate the underlying mechanism responsible for miR-495-mediated antitumor effects, we characterized the functional target gene of miR-495 in osteosarcoma cells. We found that HMGN5, a well-known oncogene (14), is a potential target of miR-495. A reduction in HMGN5 was found to inhibit proliferation and induce apoptosis of prostate cancer cells $(20,43)$, and suppression of HMGN5 induce cell cycle arrest in glioma cells (19). High expression of HMGN5 is also found in many other cancer types including renal cell carcinoma $(21)$, breast $(22)$, bladder $(23,44)$ and lung cancer (45), and it is associated with tumor progression. HMGN5 promotes tumorigenesis through regulating cyclin B1, Bcl-2 and MMP9 (20,21,23). Cyclin B1 regulates the $\mathrm{G} 2 / \mathrm{M}$ transition (46), Bcl-2 is a strong anti-apoptotic protein (47), and MMP9 promotes cancer cell metastasis (48). By promoting the expression of these genes, HMGN5 contributes to cancer development and progression. HMGN5 has been found to be highly expressed in osteosarcoma tissues associated with pathologic staging (24). Suppression of HMGN5 inhibited the invasion, induced cell cycle arrest, and promoted the sensitivity to doxorubicin-induced cell apoptosis in osteosarcoma cells (24). Suppression of HMGN5 also increased apoptosis gene expression and decreased Akt, cyclin B1 and MMP9 gene expression (24). In line with these findings, Yang et al reported that HMGN5 overexpression promoted drug resistance by upregulating autophagy in osteosarcoma cells (49). These findings indicate that HMGN5 plays an important role in osteosarcoma cells. In the present study, we demonstrated that inhibition of HMGN5 by miR-495 overexpression inhibited osteosarcoma cell proliferation and invasion, and induced cell apoptosis of osteosarcoma cells, indicating the potential for the development of a novel strategy for the treatment of osteosarcoma by targeting HMGN5. 
The epigenetic regulation of HMGN5 by miRNAs has been reported in several studies (50-52). Yao et al reported that miR-186 inhibited the growth and metastasis of bladder cancer by targeting HMGN5 (50). By targeting HMGN5, miR-340 repressed the tumorigenic potential of prostate cancer cells (51). In addition, inhibition of HMGN5 by miR-326 impeded non-small cell lung cancer cell proliferation and invasion (52). In line with our findings, these studies indicate that HMGN5 underwent epigenetic regulation during tumor progression. Targeting HMGN5 with specific miRNAs shows promise for the development of cancer therapies.

The present study found, for the first time, that miR-495 plays a critical role in osteosarcoma. Decreased levels of miR-495 were observed in osteosarcoma and miR-495 overexpression showed obvious antitumor effects. Investigation of the underlying mechanism showed that miR-495 targeted HMGN5 and inhibited HMGN5 expression. The present study provides novel insights into the molecular pathogenesis of osteosarcoma and suggests a potential molecular target for the development of miRNA-based therapy for osteosarcoma.

\section{References}

1. Botter SM, Neri D and Fuchs B: Recent advances in osteosarcoma. Curr Opin Pharmacol 16: 15-23, 2014.

2. Meyers PA, Schwartz CL, Krailo MD, Healey JH, Bernstein ML, Betcher D, Ferguson WS, Gebhardt MC, Goorin AM, Harris M, et al; Children's Oncology Group: Osteosarcoma: The addition of muramyl tripeptide to chemotherapy improves overall survival - a report from the Children's Oncology Group. J Clin Oncol 26: 633-638, 2008.

3. Hughes DP: Strategies for the targeted delivery of therapeutics for osteosarcoma. Expert Opin Drug Deliv 6: 1311-1321, 2009.

4. Bartel DP: MicroRNAs: Genomics, biogenesis, mechanism, and function. Cell 116: 281-297, 2004.

5. Winter J, Jung S, Keller S, Gregory RI and Diederichs S: Many roads to maturity: MicroRNA biogenesis pathways and their regulation. Nat Cell Biol 11: 228-234, 2009.

6. Thomson DW, Bracken CP and Goodall GJ: Experimental strategies for microRNA target identification. Nucleic Acids Res 39: 6845-6853, 2011.

7. Manikandan J, Aarthi JJ, Kumar SD and Pushparaj PN: Oncomirs: The potential role of non-coding microRNAs in understanding cancer. Bioinformation 2: 330-334, 2008.

8. Pang JC, Kwok WK, Chen Z and Ng HK: Oncogenic role of microRNAs in brain tumors. Acta Neuropathol 117: 599-611, 2009.

9. Silber J, James CD and Hodgson JG: microRNAs in gliomas: Small regulators of a big problem. Neuromolecular Med 11: 208-222, 2009.

10. Kushlinskii NE, Fridman MV and Braga EA: Molecular mechanisms and microRNAs in osteosarcoma pathogenesis. Biochemistry 81: 315-328, 2016.

11. Zhang J, Yan YG, Wang C, Zhang SJ, Yu XH and Wang WJ: MicroRNAs in osteosarcoma. Clin Chim Acta 444: 9-17, 2015.

12. Sampson VB, Yoo S, Kumar A, Vetter NS and Kolb EA: MicroRNAs and potential targets in osteosarcoma: Review. Front Pediatr 3: 69, 2015. Review.

13. Rochman M, Malicet $\mathrm{C}$ and Bustin M: HMGN5/NSBP1: A new member of the HMGN protein family that affects chromatin structure and function. Biochim Biophys Acta 1799: 86-92, 2010.

14. Shi Z, Tang R, Wu D and Sun X: Research advances in HMGN5 and cancer. Tumour Biol 37: 1531-1539, 2016.

15. Hock R, Furusawa T, Ueda T and Bustin M: HMG chromosomal proteins in development and disease. Trends Cell Biol 17: 72-79, 2007.

16. Shirakawa H, Landsman D, Postnikov YV and Bustin M: NBP-45, a novel nucleosomal binding protein with a tissue-specific and developmentally regulated expression. J Biol Chem 275: 6368-6374, 2000.

17. King LM and Francomano CA: Characterization of a human gene encoding nucleosomal binding protein NSBP1. Genomics 71: $163-173,2001$.
18. Postnikov Y and Bustin M: Regulation of chromatin structure and function by HMGN proteins. Biochim Biophys Acta 1799: 62-68, 2010.

19. Qu J, Yan R, Chen J, Xu T, Zhou J, Wang M, Chen C, Yan Y and Lu Y: HMGN5: A potential oncogene in gliomas. J Neurooncol 104: 729-736, 2011.

20. Jiang N, Zhou LQ and Zhang XY: Downregulation of the nucleosome-binding protein 1 (NSBP1) gene can inhibit the in vitro and in vivo proliferation of prostate cancer cells. Asian J Androl 12: 709-717, 2010.

21. Ji SQ, Yao L, Zhang XY, Li XS and Zhou LQ: Knockdown of the nucleosome binding protein 1 inhibits the growth and invasion of clear cell renal cell carcinoma cells in vitro and in vivo. J Exp Clin Cancer Res 31: 22, 2012.

22. Weng M, Song F, Chen J, Wu J, Qin J, Jin T and Xu J: The high-mobility group nucleosome-binding domain 5 is highly expressed in breast cancer and promotes the proliferation and invasion of breast cancer cells. Tumour Biol 36: 959-966, 2015.

23. Wahafu W, He ZS, Zhang XY, Zhang CJ, Yao K, Hao H, Song G, He Q, Li XS and Zhou LQ: The nucleosome binding protein NSBP1 is highly expressed in human bladder cancer and promotes the proliferation and invasion of bladder cancer cells. Tumour Biol 32: 931-939, 2011.

24. Zhou X, Yuan B, Yuan W, Wang C, Gao R and Wang J: The expression and clinical significance of high mobility group nucleosome binding domain 5 in human osteosarcoma. Tumour Biol 35: 6539-6547, 2014.

25. Chu H, Chen X, Wang H, Du Y, Wang Y, Zang W, Li P, Li J, Chang J, Zhao G, et al: MiR-495 regulates proliferation and migration in NSCLC by targeting MTA3. Tumour Biol 35: 3487-3494, 2014.

26. Cao M, Nie W, Li J, Zhang Y, Yan X, Guan X, Chen X, Zen K, Zhang CY, Jiang X, et al: MicroRNA-495 induces breast cancer cell migration by targeting JAM-A. Protein Cell 5: 862-872, 2014.

27. Wang L, Liu JL, Yu L, Liu XX, Wu HM, Lei FY, Wu S and Wang X: Downregulated miR-495 [corrected] inhibits the G1-S phase transition by targeting Bmi-1 in breast cancer. Medicine 94: e718, 2015.

28. Li JZ, Wang ZL, Xu WH, Li Q, Gao L and Wang ZM: MicroRNA-495 regulates migration and invasion in prostate cancer cells via targeting Akt and mTOR signaling. Cancer Invest 34: 181-188, 2016.

29. Widodo, Djati MS and Rifa'i M: Role of MicroRNAs in carcinogenesis that potential for biomarker of endometrial cancer. Ann Med Surg 7: 9-13, 2016.

30. Mishra S, Srivastava AK, Suman S, Kumar V and Shukla Y: Circulating miRNAs revealed as surrogate molecular signatures for the early detection of breast cancer. Cancer Lett 369: 67-75, 2015.

31. Formosa A, Markert EK, Lena AM, Italiano D, Finazzi-Agro' E, Levine AJ, Bernardini S, Garabadgiu AV, Melino G and Candi E: MicroRNAs, miR-154, miR-299-5p, miR-376a, miR-376c, miR-377, miR-381, miR-487b, miR-485-3p, miR-495 and miR$654-3 p$, mapped to the $14 q 32.31$ locus, regulate proliferation, apoptosis, migration and invasion in metastatic prostate cancer cells. Oncogene 33: 5173-5182, 2014.

32. Jiang X, Huang H, Li Z, He C, Li Y, Chen P, Gurbuxani S, Arnovitz S, Hong GM, Price C, et al: miR-495 is a tumorsuppressor microRNA down-regulated in $M L L$-rearranged leukemia. Proc Natl Acad Sci USA 109: 19397-19402, 2012.

33. Li Z, Cao Y, Jie Z, Liu Y, Li Y, Li J, Zhu G, Liu Z, Tu Y, Peng G, et al: miR-495 and miR-551a inhibit the migration and invasion of human gastric cancer cells by directly interacting with $P R L-3$. Cancer Lett 323: 41-47, 2012.

34. Li Z, Zhang G, Li D, Jie Z, Chen H, Xiong J, Liu Y, Cao Y, Jiang M, Le Z, et al: Methylation-associated silencing of miR-495 inhibit the migration and invasion of human gastric cancer cells by directly targeting PRL-3. Biochem Biophys Res Commun 456: 344-350, 2015

35. Chen SM, Chen HC, Chen SJ, Huang CY, Chen PY, Wu TW, Feng LY, Tsai HC, Lui TN, Hsueh C, et al: MicroRNA-495 inhibits proliferation of glioblastoma multiforme cells by downregulating cyclin-dependent kinase 6. World J Surg Oncol 11: 87, 2013.

36. Nie S, Li K, Huang Y, Hu Q, Gao X and Jie S: miR-495 mediates metabolic shift in glioma cells via targeting Glut1. J Craniofac Surg 26: e155-e158, 2015.

37. Wang C, Yun Z, Zhao T, Liu X and Ma X: MiR-495 is a predictive biomarker that downregulates GFI1 expression in medulloblastoma. Cell Physiol Biochem 36: 1430-1439, 2015. 
38. Zhang B, Yuan F, Liu J, Li Y, Zhou F, Liu X, Hao Z, Li Q, Zheng $\mathrm{Y}$ and Wang W: Hsa-miR-495 acts as a tumor suppressor gene in glioma via the negative regulation of MYB. Mol Med Rep 14: 977-982, 2016.

39. Song L, Li Y, Li W, Wu S and Li Z: miR-495 enhances the sensitivity of non-small cell lung cancer cells to platinum by modulation of copper-transporting P-type adenosine triphosphatase A (ATP7A). J Cell Biochem 115: 1234-1242, 2014

40. Xu YY, Tian J, Hao Q and Yin LR: MicroRNA-495 downregulates FOXC1 expression to suppress cell growth and migration in endometrial cancer. Tumour Biol 37: 239-251, 2016.

41. Hwang-Verslues WW, Chang PH, Wei PC, Yang CY, Huang CK, Kuo WH, Shew JY, Chang KJ, Lee EY and Lee WH: miR-495 is upregulated by E12/E47 in breast cancer stem cells, and promotes oncogenesis and hypoxia resistance via downregulation of E-cadherin and REDD1. Oncogene 30: 2463-2474, 2011.

42. Lee SH, Jung YD, Choi YS and Lee YM: Targeting of RUNX3 by miR-130a and miR-495 cooperatively increases cell proliferation and tumor angiogenesis in gastric cancer cells. Oncotarget 6: 33269-33278, 2015.

43. Zhang XY, Guo ZQ, Ji SQ, Zhang M, Jiang N, Li XS and Zhou LQ: Small interfering RNA targeting HMGN5 induces apoptosis via modulation of a mitochondrial pathway and $\mathrm{Bcl}-2$ family proteins in prostate cancer cells. Asian J Androl 14 487-492, 2012

44. Gan Y, Tan J, Yang J, Zhou Y, Dai Y, He L, Yao K and Tang Y: Knockdown of HMGN5 suppresses the viability and invasion of human urothelial bladder cancer 5637 cells in vitro and in vivo. Med Oncol 32: 136, 2015.

45. Chen P, Wang XL, Ma ZS, Xu Z, Jia B, Ren J, Hu YX, Zhang QH, Ma TG, Yan BD, et al: Knockdown of HMGN5 expression by RNA interference induces cell cycle arrest in human lung cancer cells. Asian Pac J Cancer Prev 13: 3223-3228, 2012.
46. Norbury $C$ and Nurse P: Animal cell cycles and their control. Annu Rev Biochem 61: 441-470, 1992.

47. Aizawa K, Ueki K, Suzuki S, Yabusaki H, Kanda T, Nishimaki T, Suzuki T and Hatakeyama K: Apoptosis and Bbcl-2 expression in gastric carcinomas: Correlation withclinicopathological variables, p53 expression, cell proliferation and prognosis. Int J Oncol 14: 85-91, 1999.

48. Belotti D, Paganoni P, Manenti L, Garofalo A, Marchini S, Taraboletti G and Giavazzi R: Matrix metalloproteinases (MMP9 and MMP2) induce the release of vascular endothelial growth factor (VEGF) by ovarian carcinoma cells: Implications for ascites formation. Cancer Res 63: 5224-5229, 2003.

49. Yang C, Gao R, Wang J, Yuan W, Wang C and Zhou X: High-mobility group nucleosome-binding domain 5 increases drug resistance in osteosarcoma through upregulating autophagy. Tumour Biol 35: 6357-6363, 2014.

50. Yao K, He L, Gan Y,Zeng Q, Dai Y and Tan J: MiR-186 suppresses the growth and metastasis of bladder cancer by targeting NSBP1. Diagn Pathol 10: 146, 2015.

51. Wei P, Qiao B, Li Q, Han X, Zhang H, Huo Q and Sun J: microRNA-340 suppresses tumorigenic potential of prostate cancer cells by targeting high-mobility group nucleosome-binding domain 5. DNA Cell Biol 35: 33-43, 2016.

52. Li D, Du X, Liu A and Li P: Suppression of nucleosome-binding protein 1 by miR-326 impedes cell proliferation and invasion in non-small cell lung cancer cells. Oncol Rep 35: 1117-1124, 2016. 